Artículo Original/ Original Article

http://dx.doi.org/10.18004/mem.iics/1812-9528/2021.019.01.18

\title{
Asociación entre gastos de bolsillo de salud y pobreza utilizando datos de la Encuesta Permanente de Hogares en Paraguay en el 2014
}

\author{
Edgar Giménez Caballero ${ }^{1,2}$ (D), Laura Flores ${ }^{2}$ (iD), José Carlos Rodríguez² (D), Marcelo \\ Amarilla $^{2}$ iD , Andrés Britez ${ }^{2}$ iD , Néstor Peralta ${ }^{\text {iD }}$ \\ ${ }^{1}$ Universidad Nacional de Concepción, Facultad de Medicina. Concepción, Paraguay \\ ${ }^{2}$ Investigación para el Desarrollo. Paraguay
}

Cómo referenciar este artículo/

How to reference this article:
Giménez E, Amarilla M, Brítez A, Rodríguez J.C., Flores L, Peralta N. Asociación entre gastos de bolsillo de salud y pobreza utilizando datos de la Encuesta Permanente de Hogares en Paraguay en el 2014. Mem. Inst. Investig. Cienc. Salud. 2021; 19(1): 18-30

\section{RE S U M E N}

La cobertura y acceso universal de salud requiere de protección financiera, lo que puede evaluarse a través del gasto de bolsillo ante una enfermedad. El objetivo del trabajo fue analizar la asociación entre el gasto de bolsillo de salud y la pobreza en Paraguay. Se utilizó la Encuesta Permanente de Hogares del 2014 para determinar la razón entre gasto de bolsillo ante una enfermedad con los ingresos del hogar, con las transferencias estatales para pobres, con la canasta básica de consumo y de alimentos. Fueron incluidos 5.165 hogares de los cuales $21,49 \%$ eran pobres. Hubo gasto de bolsillo en el $45,19 \%$ de los hogares asociándose significativamente con la pobreza (OR: 1,8; IC95\%: 1,57 a 2,06). El 1,99\% de los hogares tuvo gasto de bolsillo mayor a $25 \%$ de sus ingresos y el $5,98 \%$ mayor a $10 \%$ de sus ingresos, ambos indicadores se asociaron significativamente con la pobreza (OR: 3,70; IC95\%: 2,50 a 5,50 y OR: 3,04; IC95\%: 2,40 a 2,06, respectivamente). Hubo empobrecimiento en el $1,44 \%$ de los hogares, y la brecha de la pobreza pasó de $34,58 \%$ a $37,67 \%$. Entre los hogares pobres el gasto de bolsillo fue equivalente a 4,9 días de consumo, a 11,5 días de alimentación y a $42 \%$ del valor de transferencias estatales. Estos hallazgos reflejan una insuficiente protección financiera del sistema de salud en Paraguay.

Palabras clave: Gasto de bolsillo en salud, cobertura universal de salud, pobreza.

\section{Association between out-of-pocket health expenses and poverty using data from the Permanent Household Survey in Paraguay in 2014}

\begin{abstract}
Universal health coverage and access requires financial protection, which can be assessed through out-of-pocket costs for illness. The objective of the study was to analyze the association between out-of-pocket health spending and poverty in Paraguay. The 2014 Permanent Household Survey was used to determine the ratio between out-of-pocket expenses for illness and household income, state transfers for the poor, and the basic consumption and food basket. Five thousand one hundred sixty five households were included, of which $21.49 \%$ were poor. There was out-ofpocket spending in $45.19 \%$ of the households, significantly associated with poverty
\end{abstract}

Fecha de recepción: Febrero 2021. Fecha de aceptación: Abril 2021

*Autor correspondiente: Edgar Giménez Caballero. Universidad Nacional de Concepción, Facultad de Medicina. Concepción, Paraguay

Email: edgica@gmail.com 
(OR: $1.8 ; 95 \%$ CI: 1.57 to 2.06 ), $1.99 \%$ of households had out-of-pocket expenses greater than $25 \%$ of their income and $5.98 \%$ greater than $10 \%$ of their income, both indicators were significantly associated with poverty (OR: $3.70 ; 95 \% \mathrm{CI}: 2.50$ to 5.50 and OR: $3.04 ; 95 \% \mathrm{CI}: 2.40$ to 2.06 , respectively). There was impoverishment in $1.44 \%$ of households, and the poverty gap went from $34.58 \%$ to $37.67 \%$. Among poor households, out-of-pocket spending was equivalent to 4.9 days of consumption, 11.5 days of food, and $42 \%$ of the value of state transfers. These findings reflect insufficient financial protection for the health system in Paraguay.

Keywords: Out-of-pocket health expenditure, universal health coverage, poverty.

\section{INTRODUCCIÓN}

Poner fin a la pobreza, acabar con el hambre, lograr salud y bienestar son parte de los objetivos de desarrollo sostenible (ODS). Una meta es lograr cobertura universal de la salud (CUS) en $2030^{(1)}$, como parte del derecho humano a la salud con protección financiera para recibir atención ante una enfermedad ${ }^{(2)}$. Existen barreras económicas que obstaculizan este derecho, limitando el acceso universal al sistema de salud (AUS). Cuanto mayor sea la participación del gasto de bolsillo de salud (GBS) en el financiamiento sanitario, menor será la equidad, menor la protección financiera ${ }^{(3)}$ y más difícil lograr CUS y AUS. Dos formas de GBS excesivos son los gastos catastróficos de salud (GCS) y los gastos empobrecedores de salud (GES) ${ }^{(4)}$. La distribución de este gasto no es aleatoria y puede asociarse con ciertos factores sociales y económicos ${ }^{(5)}$ del mismo modo que actúan los determinantes sociales de la salud $^{(6)}$. La pobreza es uno de estos determinantes sobre la distribución de enfermedad, acceso a servicios de salud y $\operatorname{GBS}^{(7)}$ y, por otra parte, la enfermedad y Ios GBS pueden ocasionar empobrecimiento ${ }^{(8)}$.

Una estrategia integradora sobre equidad, universalidad y derecho a la salud en América Latina ha sido la atención primaria de la salud (APS $)^{(9,10)}$. Pero, puesto que la CUS se refiere a un conjunto de prestaciones y a la protección financiera, se ha planteado que estos límites pueden ser restrictivos sobre el derecho que proclama ${ }^{(11)}$ y se lo contrasta con otros modelos universales, sobre todo ante los procesos de ajustes o reformas a los sistemas de salud emprendidos en esta región ${ }^{(12)}$.

En el marco de estos procesos de ajustes y reformas, varios estudios en países latinoamericanos han demostrado la asociación entre determinantes de la salud y la distribución del GBS. Perticara y colaboradores, compararon el GBS entre siete países latinoamericanos encontrando tasas de empobrecimiento entre el $1 \%$ y $1,9 \%$ debido a gastos médicos asociados a diferentes determinantes ${ }^{(13)}$. En otro estudio, Knaul y colaboradores compararon GCS y GES en doce países latinoamericanos, concluyendo que algunos grupos fueron más vulnerables de enfrentar gastos excesivos ya sea debido a sus ingresos o por la estructura del hogar ${ }^{(14)}$. En un estudio sobre Argentina, Albedaño concluyó que el GCS estuvo presente en el 2,3\% de los hogares y GES en $1,7 \%$ de los hogares y que la distribución del GBS estuvo asociado a ciertos determinantes sociales ${ }^{(15)}$. En Brasil, Luiza encontró GCS en el 5,3\% de los hogares (16). En Chile, gallegos y Muñoz encontraron una proporción de hogares con GCS de $6 \%{ }^{(17)}$. Otro estudio, de Koch y colaboradores, en el marco de la reforma de salud chileno, concluyó que a pesar de los avances en protección financiera, los esfuerzos han sido insuficientes para proteger a los grupos más pobres de $\mathrm{GBS}^{(18)}$. En Colombia, Pérez y Silva reportaron que el GCS afectó al 4,1\% de los hogares distribuidos según ciertos determinantes y regiones del país ${ }^{(19)}$. Otro estudio específico sobre hogares de Cartagena, Colombia, de Alvis-Zakzuk y colaboradores, encontró diferencias entre la incidencia del GBS sobre los ingresos y la distribución de GCS entre hogares pobres y no pobres: el GBS fue el $30 \%$ de los ingresos de hogares pobres y el $7 \%$ de los hogares ricos, mientras que el riesgo de GCS fue 30,6\% en los hogares pobres y de $8,6 \%$ en los hogares de mayor ingreso ${ }^{(20)}$. En México, Knaul y colaboradores encontraron asociación significativa ente la afiliación al seguro popular de salud y la reducción de GES ${ }^{(21)}$. Un estudio de Proaño sobre Perú, concluyó que el GCS se asoció a la pobreza, al área rural, al tamaño del hogar, a la presencia de adultos mayores y a enfermedades crónicas ${ }^{(22)}$ mientras que otro estudio de Pavone y Sánchez encontró que el GBS entre los pobres se asoció con no estar afiliado al Seguro Integral de 
Salud, con el nivel de complejidad de la atención y con ser adulto mayor ${ }^{(23)}$. En Perú, Guerrero encontró $1,3 \%$ de hogares con GCS y $1,4 \%$ con GES y que el GBS de los hogares no pobres fue 8,2 veces más que el de un hogar pobre extremo y 3,9 veces más que un hogar pobre no extremo ${ }^{(24)}$.

Estos resultados no son estáticos en el tiempo. En general, estudios como éstos utilizan como fuente de datos las encuestas de hogares ${ }^{(25)}$ aplicando diferentes metodologías para definir GBS excesivos en función de los ingresos, de los gastos, del consumo 0 de la capacidad de pago de las personas 0 de los hogares ${ }^{(4,5,8,25)}$. En el ámbito de los ODS se definieron indicadores estandarizados de GBS en relación a los ingresos y a parámetros sobre pobreza monetaria para dar seguimiento a los avances de protección financiera en la CUS ${ }^{(26-28)}$.

En el caso del Paraguay, el GBS es una de las principales fuentes de financiamiento sanitario ${ }^{(29-31)}$ y algunos estudios han medido $\operatorname{GCS}^{(32-34)}$ y $\operatorname{GES}^{(34,35)}$, siendo el principal componente la compra de medicamentos ${ }^{(36,37)}$. La pobreza se ha asociado a una mayor incidencia del GBS sobre los ingresos del hogar ${ }^{(33)}$ y a una mayor proporción de gastos por medicamentos ${ }^{(36)}$. En Paraguay la pobreza se mide en términos monetarios en función al costo de una canasta básica de consumo cuyos precios se actualizan periódicamente con base a la Encuesta de Ingresos y Gastos (EIG). Esta incluye la canasta básica de alimentos cuyo monto define a la línea de pobreza extrema. Los datos se actualizan anualmente a partir de la Encuesta Permanente de Hogares $(E P H)^{(38)}$. El país cuenta con políticas para reducir la pobreza, entre ellas los subsidios o transferencia estatales ${ }^{(39,40)}$. La inclusión de los pobres al sistema de salud paraguayo es a través de los servicios dependientes del Ministerio de Salud Pública y Bienestar Social (MSPBS). El derecho a la salud y la equidad está reconocido en el marco legal( ${ }^{(41,42)}$. Para evitar barreras económicas de acceso a los pobres, está vigente una ley de gratuidad de aranceles de las prestaciones del MSPBS ${ }^{(43)}$. Sin embargo, los procesos de cumplimiento de estas leyes son insuficientes para lograr un sistema de protección social efectivo ${ }^{(44,45)}$.

Este estudio analiza cómo afecta el GBS a los hogares pobres de Paraguay y qué tanto está protegidos para evitar catástrofes financieras ante una enfermedad. Los resultados serán indicativos de las brechas en el momento de partida ante los desafíos para lograr CUS en los ODS y podrán ser útiles para evaluar los progresos.

\section{MATERIAL Y MÉTODOS}

Corresponde a un estudio transversal a partir del análisis de la base de datos de la Encuesta Permanente de Hogares (EPH) del Paraguay del año 2014 (último año disponible sobre gastos de bolsillo de salud). El diseño muestral de esta encuesta fue probabilístico, por conglomerados, con probabilidad proporcional al tamaño, bietápico y estratificado con una representación nacional. El tamaño de la muestra de la EPH en 2014 fue de 5.165 hogares.

A partir de esta base de datos se estudiaron las siguientes variables independientes:

(1) Hogares con enfermos o accidentados: hogares con al menos un integrante enfermo o accidentado en los últimos 90 días. (2) Hogares con personas que consultan: hogares con al menos una persona enferma 0 accidentada que consultó con un profesional médico en los últimos 90 días. (3) Hogares con gasto de bolsillo de salud: hogares con gasto mayor a cero en gasto de bolsillo de salud (GBS), contemplando las categorías de gastos de consulta, medicamentos, exámenes y hospitalización, durante los últimos 90 días. (4) Hogares con medicamentos gratuitos: hogares que recibieron medicamentos en forma gratuita, durante los últimos 90 días. (5) Magnitud del gasto de bolsillo de salud (GBS): sumatoria del GBS para cada hogar en los últimos 90 días. Se expresó en guaraníes (Gs.) corrientes para el 2014. Se calculó de forma desagregada el gasto de bolsillo por medicamentos (GBSm), el cual está incluido en el cálculo de GBS. (6) Magnitud del ingreso mensual del hogar: sumatoria de los diferentes ingresos de cada hogar durante un mes, provenientes de la ocupación principal, de la ocupación secundaria y otras ocupaciones (si los hubiera), ingresos por alquileres o rentas, por jubilaciones o 
pensiones, transferencias familiares regulares en dinero provenientes del país y del exterior, prestaciones por divorcios, transferencias monetarias y no monetarias del gobierno y otros ingresos. Se expresó en guaraníes (Gs.) corrientes. Se desagregaron los ingresos por transferencias estatales para combatir la pobreza, los cuales fueron incluidos en el cálculo del ingreso mensual total del hogar ${ }^{(7)}$. Razón GBS/Ingreso: razón entre la magnitud del GBS y el ingreso de los hogares. Puesto que el GBS fue trimestral, también los ingresos fueron trimestrales. Se realizó un cálculo específico para el GBSm y para los ingresos por transferencias estatales de los programas Tekoporá y de pensión alimentaria para adultos mayores ${ }^{(8)}$. GBS excesivo: hogares según hayan tenido una razón GBS/ingreso mayor a 0,1. Entre estos hogares se calculó aquellos con una razón GBS/ingreso mayor a 0,25 considerando a estos como gastos catastróficos de salud (GCS). La proporción de hogares con este tipo de gastos se expresó en porcentaje sobre todos los hogares. La proporción de hogares con una razón GBS/ingreso mayor a 0,25 fueron incluidos en el \% de hogares con una razón GBS/ingreso mayor a $0,1^{(9)}$. Razón GBS/valor de la canasta básica: razón entre la magnitud del GBS y el valor diario de la canasta básica de consumo y de la canasta básica de alimentos para cada hogar. Se utilizó el valor oficial para un mes para una persona, considerando la cantidad de integrantes del hogar, dividiéndolo entre 30 días. Para esta razón se consideró solamente a aquellos hogares que tuvieron GBS $^{(10)}$ GBS empobrecedor o empobrecimiento por GBS: Se calculó de dos maneras: a) hogares según que la diferencia entre sus ingresos trimestrales y el GBS sea menor o no a la línea de pobreza y pobreza extrema. b) Variación de la brecha de la pobreza definida como la variación del porcentaje de ingreso requerido para alcanzar la línea de pobreza, después de este gasto. Se utilizó la definición oficial de pobreza como aquel conjunto de personas residentes en hogares cuyo nivel de gasto (medido a través del ingreso) es inferior al costo de una canasta básica de consumo constituida por el conjunto de bienes y servicios que satisfacen ciertos requerimientos mínimos, tanto alimentarios como no alimentarios. En el 2014, en valores corrientes, la línea de pobreza había sido definida en 626.159 guaraníes para el área metropolitana, y 447.758 guaraníes para el resto urbano y en 386.388 guaraníes para el área rural.

Se aplicó pruebas de significancia estadística para buscar asociación entre las variables independientes y la condición de pobreza con la prueba de $\mathrm{Chi}^{2}$ para las variables cualitativas y Mann Whitney para las cuantitativas. En todos los casos se consideró un nivel de significancia de 0,05 . También se calculó el odds ratio (OR) con su intervalo de confianza de $95 \%$.

Los resultados se presentaron para valores de la muestra y para la estimación de los hogares que representan. El factor de expansión utilizado fue una variable que ajusta las observaciones muestrales de la EPH a las proyecciones poblacionales del censo 2012. Esta variable está disponible en la base de datos de la EPH bajo la denominación de "fex" para el factor de expansión de hogares y de "facpob" para el factor de expansión de población en la página del Instituto Nacional de Estadística (INE) ${ }^{(46)}$.

\section{RESULTADOS}

Para el estudio fueron incluidos los 5.165 hogares de la muestra de la EPH del 2014. Esta muestra fue representativa de 1.621 .525 hogares a nivel nacional. Cada hogar estaba integrado por 4,03 personas en promedio y con las proyecciones censales se estimó una población total de 6.546.971.

Del total de la muestra, $2.334(45,19 \%)$ hogares tuvieron GBS y entre estos 309 hogares (5,98\%) presentaron GBS excesivos, mayor al $10 \%$ de sus ingresos. Entre estos, 103 hogares $(1,99 \%$ de la muestra) tuvieron GBS mayor al $25 \%$ de sus ingresos (Figura 1). 
Fuente: Elaboración propia a partir de datos de la Encuesta Permanente de Hogares $(\mathrm{EPH}) 2014$.

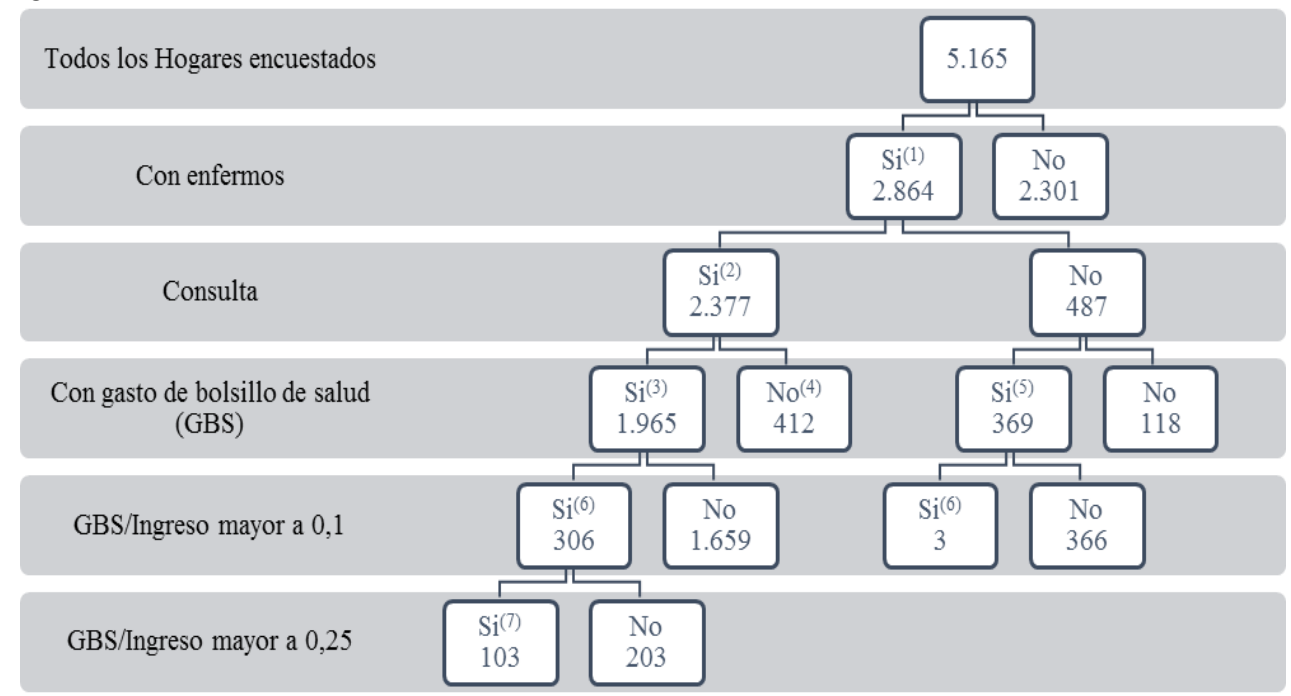

Figura 1: Distribución de hogares para el estudio del gasto de bolsillo de salud en la encuesta permanente de hogares (EPH) del Paraguay en el año 2014.

(1) Corresponde a todos los hogares con enfermos o accidentados durante los últimos 90 días previos a la encuesta. Si una misma persona del hogar estuvo enferma en más de una oportunidad, se registra sólo la última vez. No se registran enfermos crónicos que están en tratamiento salvo que tengan algún evento nuevo de enfermedad o accidente en ese tiempo.

(2) Se consideró que sí consultó cuando lo hizo con un profesional médico.

(3) Se incluyó como gasto de bolsillo de salud (GBS) los gastos por consulta, medicamentos, exámenes y hospitalización. Quedaron excluidos gastos de transporte, alimentos y otros.

(4) Dado que consultaron y no tuvieron GBS puede ser: a) debido a que están cubiertos totalmente; b) no accedieron

(5) Dado que no consultaron y sí tuvieron GBS este gasto puede ser debido a que: a) se automedicaron; b) consultaron con un profesional no médico; c) consultaron en el sector de medicina tradicional.

(6) Se consideró GBS excesivo cundo la razón GBS/ingreso (del hogar) fue mayor a 0,1 .

(7) Entre los hogares con gastos excesivos, se calculó aquellos cuya razón GBS/ingreso fue mayor a 0,25.

Aplicando expansores censales se estimó que la cantidad de hogares afectados a nivel nacional eran $88.531(5,46 \%)$ hogares con GBS mayor a $10 \%$ de los ingresos y $28.023(1,73 \%)$ hogares con GBS mayor a $25 \%$ de los ingresos. La población afectada en ellas fue $347.315(5,30 \%)$ y $110.942(1,69 \%)$, respectivamente según estos indicadores.

En la muestra, el $21,49 \%$ de los hogares eran pobres y el $78,51 \%$ eran no pobres. Considerando la situación de pobreza, la proporción de hogares con enfermos, que consultaron con un profesional médico, que tuvieron acceso a medicamentos gratuitos, que presentaron GBS y GBS excesivo fue significativamente mayor entre los hogares pobres. Por cada hogar no pobre con GBS y GBSm hubo 1,8 hogares pobres con GBS y con GBSm. Además, por cada hogar no pobre con GBS excesivo hubo 3,04 hogares pobres con este tipo de gasto (Tabla 1 ). 
Tabla 1: Paraguay 2014. Distribución de hogares con al menos un enfermo o accidentado, que consulta con un médico, con gasto de bolsillo de salud (GBS), gasto de bolsillo por medicamentos (GBSm), con acceso a medicamentos gratuitos y con GBS excesivos.

\begin{tabular}{|l|c|c|c|c|}
\hline Variables & $\begin{array}{c}\text { Pobres (\%) } \\
\mathbf{n = 1 . 1 1 0}\end{array}$ & $\begin{array}{c}\text { No pobres (\%) } \\
\mathbf{n = 4 . 0 5 5}\end{array}$ & Valor p & OR (IC95\%) \\
\hline Con enfermos & 69,55 & 51,59 & $<0,001$ & $2,14(1,85$ a 2,47$)$ \\
\hline Consultan con médico & 56,49 & 43,16 & $<0,001$ & $1,71(1,49$ a 1,96$)$ \\
\hline Medicamento gratis & 33,87 & 22,86 & $<0,001$ & $1,73(1,49$ a 1,99$)$ \\
\hline Tienen GBS & 56,67 & 42,05 & $<0,001$ & $1,80(1,57$ a 2,06$)$ \\
\hline Tienen GBSm & 55,41 & 40,81 & $<0,001$ & $1,80(1,57$ a 2,06$)$ \\
\hline GBS/Ingreso>0,1 & 12,07 & 4,32 & $<0,001$ & $3,04(2,40$ a 3,86$)$ \\
\hline GBS/Ingreso>0,25 & 4,59 & 1,28 & $<0,001$ & $3,07(2,50$ a 5,50$)$ \\
\hline
\end{tabular}

Fuente: Elaboración propia a partir de datos de la Encuesta Permanente de Hogares (EPH) 2014.

(*) Todos los datos se refieren a los últimos 90 días previos a la encuesta. Por lo tanto también los ingresos del hogar se han trimestralizado.

También la magnitud del GBS se asoció de forma significativa con la pobreza. En tanto que la cantidad gastada ante la enfermedad fue significativamente mayor en los hogares no pobres, la razón de este gasto con los ingresos fue significativamente mayor para los pobres (Tabla 2).

Entre los hogares que tuvieron GBS, este gasto fue equivalente a 8,48 días del valor de una canasta de consumo básica (canasta estándar, establecida en el ámbito gubernamental que define la línea de pobreza) y a 20,33 días del valor de una canasta básica de alimentos (canasta estándar, establecida en el ámbito gubernamental que define la línea de pobreza extrema). Considerando los hogares pobres, para quienes el valor de estas canastas es más aproximado, el GBS fue equivalente a 4,95 días de consumo básico y a 11,47 días de alimentación. Para los hogares no pobres el GBS fue equivalente a 9,76 días del valor de la misma canasta básica de consumo y a 23,56 días del valor de la misma canasta básica de alimentos (Tabla 2).

Entre los hogares pobres, el $17,07 \%$ recibía transferencias estatales con un promedio de 280.834 Gs. También entre los no pobres hubo $7,71 \%$ hogares que recibía transferencias estatales con un promedio de 383.803 Gs. La razón del GBS sobre estas transferencias fue 0,39 para los pobres y 0,44 para los hogares no pobres (Tabla 2).

Tabla 2: Paraguay 2014. Descripción de variables de gasto de bolsillo de salud (GBS), gasto del bolsillo de salud por compra de medicamentos (GBSm) y su relación con los ingresos del hogar en un trimestre, sobre el valor de la canasta diaria consumo, de la canasta básica de alimentos, y de la razón del GBS sobre las transferencias estatales para combatir la pobreza.

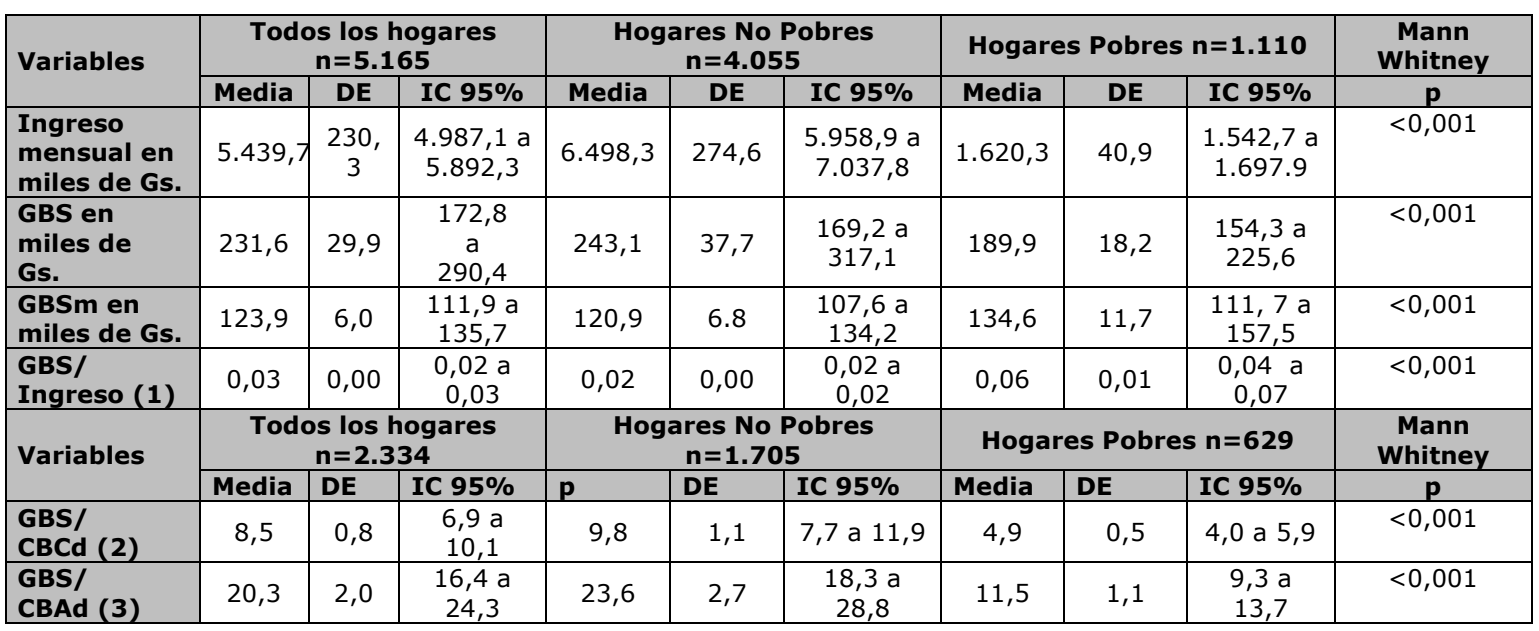




\begin{tabular}{|c|c|c|c|c|c|c|c|c|c|c|}
\hline \multirow[t]{2}{*}{ Variables } & \multicolumn{3}{|c|}{$\begin{array}{c}\text { Todos los hogares que } \\
\text { reciben transferencias } \\
\text { estatales } n=523\end{array}$} & \multicolumn{3}{|c|}{$\begin{array}{c}\text { Hogares No Pobres que } \\
\text { reciben transferencias } \\
\text { estatales } n=313\end{array}$} & \multicolumn{3}{|c|}{$\begin{array}{c}\text { Hogares Pobres que } \\
\text { reciben transferencias } \\
\text { estatales } n=210\end{array}$} & \multirow{2}{*}{$\begin{array}{c}\begin{array}{c}\text { Mann } \\
\text { Whitney }\end{array} \\
\mathbf{p} \\
\end{array}$} \\
\hline & Media & DE & IC $95 \%$ & Media & DE & IC $95 \%$ & Media & DE & \begin{tabular}{|l|} 
IC95\% \\
\end{tabular} & \\
\hline $\begin{array}{l}\text { GBS/ } \\
\text { Transferenci } \\
\text { as (4) }\end{array}$ & 0,4 & 0,1 & 0,3 a 0,6 & 0,4 & 0,1 & 0,2 a 0,7 & 0,4 & 0,1 & 0,2 a 0,6 & 0,048 \\
\hline
\end{tabular}

Fuente: Elaboración propia a partir de datos de la Encuesta Permanente de Hogares (EPH) 2014.

(1) Todos los datos se refieren a los últimos 90 días previos a la encuesta. Por lo tanto, también los ingresos del hogar se han trimestralizado.

(2) El valor de la canasta básica de consumo (CBC) se establece oficialmente por el gobierno nacional y define la línea de pobreza en el país. El cálculo se realizó para aquellos hogares que tuvieron gasto de bolsillo de salud (GBS) en relación con el consumo del hogar por día (CBCd).

(3) El valor de la canasta básica de alimentos (CBA) se establece oficialmente por el gobierno nacional y define la línea de pobreza extrema en el país. El cálculo se realizó para aquellos hogares que tuvieron gasto de bolsillo de salud (GBS) en relación con el consumo de alimentos del hogar por día (CBAd).

(4) Corresponde a las transferencias monetarias para combatir la pobreza a través de los programas Tekoporá y de pensión alimentaria para adultos mayores pobres. Tanto el GBS como el valor de las transferencias corresponden a un trimestre.

Finalmente, se calculó el empobrecimiento debido a GBS. El resultado fue que 78 casos no pobres de la muestra quedaron por debajo de la línea de pobreza después del GBS. Estos casos representan a 23.387 hogares a nivel nacional $(1,44 \%$, de todos los hogares). Entre estos hogares, 2.925 quedaron por debajo de la línea de pobreza extrema. También los hogares pobres que tuvieron GBS profundizaron su situación de pobreza, lo que pudo evaluarse mediante la variación de la brecha de la pobreza que pasó de $34,58 \%$ a $37,67 \%$ después del GBS. Además, el $4,6 \%$ de los hogares pobres no extremos (13.104 hogares) quedaron por debajo de la línea de pobreza extrema después del GBS (Tabla 3).

Tabla 3: Paraguay 2014. Hogares en situación de pobreza y brecha de la pobreza antes del GBS y después del GBS en la muestra y la estimación de hogares que representa esa muestra aplicando un factor censal de expansión.

\begin{tabular}{|c|l|l|l|l|}
\hline \multirow{2}{*}{ Variables } & \multicolumn{2}{|l|}{ Antes del GBS } & \multicolumn{2}{l|}{ Después del GBS* } \\
\cline { 2 - 5 } & Muestra & Expandido* & Muestra & Expandido* \\
\hline $\begin{array}{c}\text { Número de hogares en situación de } \\
\text { pobreza }\end{array}$ & 1.110 & 351.914 & 1188 & 375301 \\
\hline $\begin{array}{c}\text { Número de hogares en } \\
\text { situación de pobreza no } \\
\text { extrema }\end{array}$ & 924 & 287.406 & 989 & 294.764 \\
\hline $\begin{array}{l}\text { Número de hogares en } \\
\text { situación de pobreza extrema }\end{array}$ & 186 & 64.508 & 199 & 80.537 \\
\hline Brecha de la pobreza en \% & 33,69 & 34,58 & 36,93 & 37,67 \\
\hline
\end{tabular}

Fuente: Elaboración propia a partir de datos de la Encuesta Permanente de Hogares (EPH) 2014.

* Nota: El factor de expansión utilizado es una variable que ajusta las observaciones muestrales de la EPH a

las proyecciones poblacionales del censo de población 2012. 
Fuente: Elaboración propia a partir de datos de la Encuesta Permanente de Hogares (EPH) 2014.

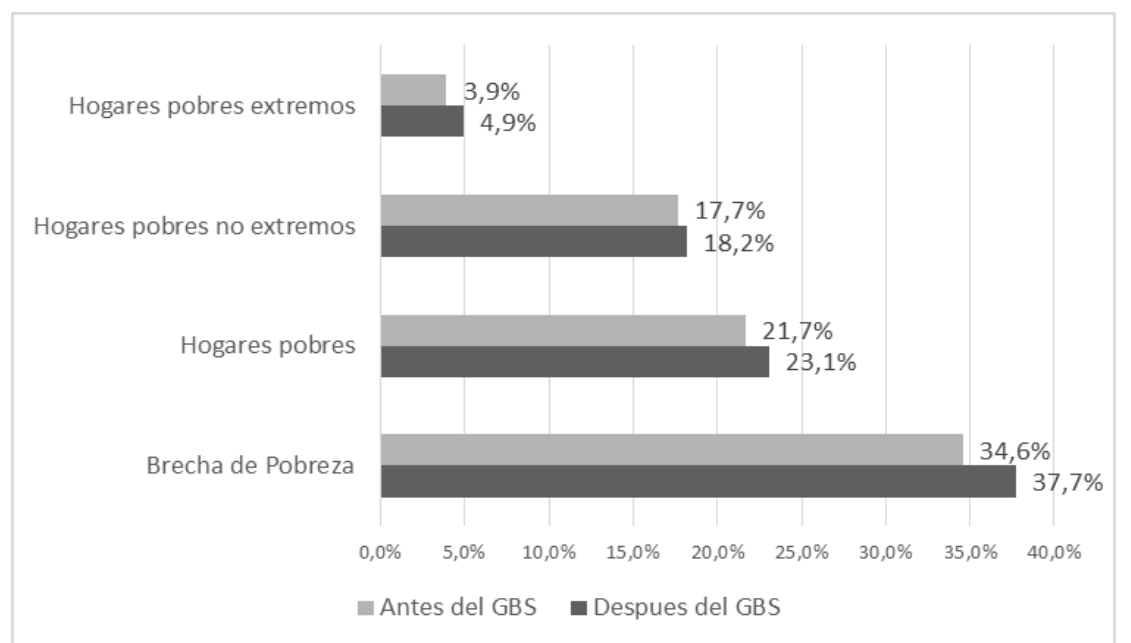

Figura 2: Distribución porcentual de hogares en situación de pobreza y brecha de la pobreza antes del GBS y después del GBS en Paraguay en el año 2014.

Nota: Se utilizan porcentajes sobre un total de 1.621 .525 hogares estimados por la DGEEC para el año 2014. GBS: gasto de bolsillo de salud

\section{DISCUSIÓN}

Los resultados de este estudio señalaron que los pobres tuvieron una mayor incidencia de GBS, lo cual estuvo determinado por una mayor ocurrencia de enfermedad y por una menor capacidad financiera para recibir atención en un sistema de salud financiado principalmente con fuentes privadas ${ }^{(29,30)}$. En efecto, la posibilidad de enfermar fue el doble para los hogares pobres puesto que por cada un hogar no pobre con enfermos había dos hogares pobres con enfermos (OR 2,14). Esto fue consistente con el concepto de determinantes sociales de la salud ${ }^{(6)}$, con las teorías sobre desigualdad y pobreza en el sector salud ${ }^{(7)}$ así como con los hallazgos en varios países de América Latina sobre GBS y ciertos determinantes sociales ${ }^{(5,13)}$. Teniendo mayor chance de enfermedad es razonable que esta situación haya derivado en una mayor necesidad de consultas (OR 1,7$)$ y en una mayor posibilidad de GBS (OR 1,8 ) en un sistema de salud donde ésta es la fuente predominante de financiamiento ${ }^{(31,34)}$ sobre todo para comprar medicamentos ${ }^{(36,37)}$. Por otra parte, los pobres dependen de un sistema de protección social no contributivo insuficientemente financiado para cubrir las necesidades ${ }^{(45)}$, están poco cubiertos ante una enfermedad ${ }^{(4)}$, supeditados a lo que se encuentra disponible en los servicios públicos, sobre todo en el Ministerio de Salud Pública y Bienestar Social. Los resultados señalaron que los pobres tuvieron más posibilidad de obtener medicamentos gratuitos $(\mathrm{OR} 1,7)$ pero que este mecanismo ${ }^{(43)}$ fue insuficiente para evitar una mayor posibilidad de GBS excesivos y catastróficos para ellos (OR 3,04 y 3,07, respectivamente). Esta distribución desigual del GBS y GCS entre los pobres fue consistente con los hallazgos en otros países como Colombia, México y Perú, donde el GBS se ha asociado a la vulnerabilidad social y a la falta de cobertura de la seguridad social ${ }^{(20,22,24)}$.

El hecho que el promedio de GBS de los no pobres fuera significativamente mayor que el de los pobres indica la existencia de un mercado de salud con precios variables y el acceso desigual al sistema de salud según la capacidad de pago de los hogares. Aunque el promedio del GBS fue menor para los pobres, la razón del gasto sobre sus ingresos fue tres veces mayor cuando se comparó con lo no pobres. Estas desigualdades en el acceso y en el efecto sobre la economía del hogar deterioran la equidad y son signos de una limitada protección financiera para este grupo vulnerable de la población.

Otro resultado fue que el GCS, correspondiente al indicador 3.8.2 de los ODS ${ }^{(26)}$, afectó al $1,99 \%$ de los hogares paraguayos. Este valor coincidió con lo publicado en el reporte global sobre protección financiera en salud ${ }^{(8)}$. El desempeño de este indicador se aproximó al valor de $2 \%$ de GCS reportado para Paraguay bajo la definición de GBS 
que supera el $40 \%$ de capacidad de pago del hogar ${ }^{(32)}$. Sin embargo, fue diferente del valor de 9,9\% para el país publicado por el Banco Mundial utilizando el mismo indicador de ODS ${ }^{(34)}$ lo que pudiera deberse a cuestiones metodológicas sobre la unidad de análisis (hogares o población), la temporalidad (mensual o trimestral) y a la definición misma del GBS (solo los gastos directamente relacionados o también los gastos indirectamente relacionados). El valor de GCS de este estudio presentó aproximaciones con los hallazgos para otros países del mismo continente. Por ejemplo, estuvo en el rango de GCS de $0,5 \%$ a $5,6 \%$ reportado por Perticara para siete países ${ }^{(13)}$; también en el rango estudiado por Knaul y col para doce países cuya incidencia de GCS estuvo entre $0,4 \%$ a $11 \%{ }^{(14)}$. Fue más aproximado a los resultados para Argentina y Perú con incidencias de GCS de $2,3 \%$ y $1,3 \%$ respectivamente ${ }^{(13,24)}$. Por otra parte, también puede compararse con incidencias más altas de GCS como los reportados para Brasil y Chile de $5,3 \%$ y $6 \%$ respectivamente ${ }^{(16,17)}$.

El indicador GBS mayor a $10 \%$ de los ingresos, que en este trabajo se consideró excesivo, afectó a $5,98 \%$ de los hogares, lo que resultó diferente a la medición del mismo indicador en el informe global de protección financiera, según el cual afectó al $7,1 \%$ de los hogares ${ }^{(8)}$.

El GBS no solo se asoció con la pobreza sino que también generó pobreza, lo que pudo verificarse mediante el empobrecimiento de hogares que antes del GBS eran no pobres y mediante la variación de la brecha de la pobreza. El GES calculado en este trabajo fue de $1,44 \%$ de todos los hogares. Este resultado se ubicó de forma intermedia a $1,66 \%$ calculado en el reporte global sobre protección financiera ${ }^{(8)}$ y a $3,1 \%$ reportado por el Banco Mundial ${ }^{(34)}$. La diferencia puede explicarse debido a que en este trabajo se utilizó la línea de pobreza definida por el valor de una canasta de consumo, en tanto que aquellos informes utilizaron una línea de pobreza internacional. Estos hallazgos para el Paraguay fueron similares a los hallazgos de Perticara para siete países latinoamericanos donde las tasas de empobrecimiento estuvieron entre $1 \%$ y $1,9 \%^{(13)}$, al de Albedaño para Argentina con GES en $1,7 \%$ de los hogares ${ }^{(15)}$ y al de Guerrero en Perú con GES en $1,4 \%$ de los hogares ${ }^{(24)}$.

En cuanto el efecto del GBS sobre el consumo, los resultados señalaron que ante una enfermedad pudo plantearse un dilema para los pobres: escoger entre gastos de salud y gastos para consumo básico, o, dicho de otro modo, el dilema de escoger entre comprar medicamentos o alimentos. Otro resultado fue que el GBS fue el equivalente al $42 \%$ de las transferencias estatales destinadas para la subsistencia de los pobres. Estos cálculos complementarios en relación al consumo y consumo esencial contribuyen a una mejor comprensión de los efectos del GBS sobre la economía de los hogares y fueron señalados en recomendaciones para estudiar GBS a partir de fuentes de datos como la EPH ${ }^{(25)}$. Utilizar las canastas básicas de consumo básico y de alimentos es una innovación para estudios de GBS en Paraguay, pues no hay antecedentes de estudios similares.

En conjunto, estos resultados señalaron la necesidad de aumentar la coordinación y sinergias entre los programas de protección social, aumentada la cobertura, tendiente a la universalización tanto del acceso a la salud como a otros derechos sociales ${ }^{(44,45)}$.

Las limitaciones de este estudio fueron los propios de los estudios transversales y de los estudios de GBS a partir de encuestas de hogares ${ }^{(25)}$. Las encuestas de hogares son útiles para analizar las características del GBS brindando una aproximación sobre su efecto económico a nivel microeconómico ${ }^{(25)}$ y permiten seguir y evaluar los indicadores de protección financiera ante los $\operatorname{ODS}^{(26,27)}$. Otra limitación es que el registro del GBS en la EPH no es exhaustivo, ya que no registra todos los gastos de una persona sino sólo el último.

En el caso del Paraguay, la última vez que se recogieron datos sobre gastos en salud en la EPH fue en 2014. Los resultados de este estudio, para ese año, pueden tomarse como línea de base con miras al 2030 para evaluar los progresos sobre CUS y protección financiera ${ }^{(8)}$ y por tanto su actualización mediante nuevas encuestas será de gran utilidad.

Hay varias cuestiones metodológicas a considerar. 
Una de ellas es la temporalidad. Se ha postulado que los datos para el cálculo de gastos catastróficos y empobrecedores deben ser mensuales ${ }^{(4)}$. En el caso de la EPH del Paraguay los datos de ingresos del hogar se refieren a un mes, en tanto que el gasto de salud a un trimestre. En esta investigación se resolvió mantener la temporalidad original del gasto de salud y trimestralizar el ingreso promedio del hogar, coincidiendo con otros estudios sobre Paraguay de los mismos autores ${ }^{(32,33)}$ y de otros autores ${ }^{(8,37)}$.

También hay que considerar las definiciones operativas de GBS y el denominador utilizado para las tasas. Estas definiciones dependerán de la pregunta de investigación. En este estudio las preguntas estuvieron enmarcadas en el desafío de lograr CUS y AES ante IOS ODS. Es por eso que se adoptó una definición de gastos directamente relacionados con la atención y tratamiento ante una enfermedad ${ }^{(4,26)}$ y se excluyó otros gastos como transporte y alimentación que fueron incluidos en otros trabajos ${ }^{(34,37)}$. En cuanto a los denominadores, se consideró el total de hogares según otras publicaciones. Los autores consideran que este denominador es el más apropiado para evaluar los cambios en función a cambios de políticas. Otros denominadores posibles para responder otras preguntas podrían ser: enfermos o enfermos que consultan.

Otra cuestión es que estos indicadores pueden referirse a hogares o a la población. La escogencia de uno u otro presenta ventajas y desventajas. En este trabajo se optó por definirlos por hogares ya que esta fue la unidad muestral ${ }^{(38)}$.

Por último, en este estudio se analizó de forma indirecta la relación del GBS con el consumo del hogar, utilizando el valor oficial de una canasta estándar de consumo y con transferencias estatales a los pobres. Este método es más apropiado apropiados para aquellos hogares pobres o para los hogares con menores ingresos en una estratificación de hogares por ingresos. Para los estratos de hogares de mayor ingreso habrá que utilizar otros valores de canastas de consumos o bien recurrir al consumo real. Pero estos datos no están disponibles. Los autores consideran que estos indicadores podrían ser complementarios para dar seguimiento al indicador 3.8.2 de los ODS ${ }^{(8,26)}$, considerando el contexto paraguayo, y que además podrían ser útiles en países con características similares.

Para futuros estudios podrían considerarse los siguientes temas en relación al GBS: a) incluir en el análisis otros determinantes sociales como la tenencia de seguro, la estructura del hogar, el área de residencia, las características del jefe de hogar; b) incidencia en otras poblaciones vulnerables; c) estudio de casos por niveles de complejidad o por grupo de enfermedad; d) incluir otros indicadores complementarios sobre el consumo e ingresos como la relación sobre salarios mínimos; e) relacionar la variación temporal del GBS con otros indicadores de financiamiento sanitario y otros indicadores económicos.

EI GBS excesivo ante una enfermedad se asoció significativamente con la pobreza en el Paraguay. Hubo hogares no pobres que se empobrecieron para utilizar servicios de salud y los hogares pobres profundizaron su situación de pobreza. Las políticas para aumentar el acceso como la distribución gratuita de medicamentos, eliminación de aranceles y transferencias estatales fueron insuficientes para brindar protección financiera a los pobres ante una enfermedad.

\section{AGRADECIMIENTOS}

Este estudio es parte del proyecto PINV18-672 financiado por el programa PROCIENCIA de CONACYT presentado por Investigación para el Desarrollo en convenio con la Facultad de Medicina de la Universidad Nacional de Concepción.

\section{REFERENCIAS BIBLIOGRÁFICAS}

1. World Health Organization. Health in 2015: from MDGs, Millennium Development Goals to SDGs, Sustainable Development Goals. Ginebra, 2015.

2. Organización de las Naciones Unidas. Consejo Económico y Social. El derecho de toda persona al disfrute del más alto nivel posible de salud física y mental. Informe del Relator Especial, Sr. Paul Hunt. Comisión de Derechos Humanos (documento E/CN.4/2005/51). Ver 
también El derecho al disfrute del más alto nivel posible de salud, observación general 14, (documento E/C.12/2000/4), presentado por el Comité de Derechos Económicos, Sociales y Culturales (ONU) durante el $22^{\circ}$ período de sesiones, Ginebra, 25 de abril a 12 de mayo de 2000.

3. OMS. Financiación de los sistemas de salud. El camino hacia la cobertura universal. Informe sobre la salud en el mundo 2010. Informe de un Grupo Científico de la OMS; Ginebra: OMS; 2010. Disponible en: http://whqlibdoc. who.int/whr/2010/978 9243564029 spa.pd

4. OMS. Reporte: metodología OMS de análisis de la equidad del financiamiento de la salud. Ginebra, OMS; 2003.

5. Knaul FM, Wong R, Arreola-Ornelas $\mathrm{H}$. Household Spending and Impoverishment. Volume 1 of Financing Health in Latin America Series. Cambridge, MA: Harvard Global Equity Initiative, in collaboration with Mexican Health Foundation and International Development Research Centre, 2012; distributed by Harvard University Press.

6. OMS. Comisión de Determinantes Sociales de la Salud. Subsanar las desigualdades en una generación. alcanzar la equidad sanitaria actuando sobre los determinantes sociales de la salud. Ginebra: OMS; 2009.

7. Wagstaff A. Pobreza y desigualdades en el sector de la salud. Rev Panam Salud Pública. 2002; 11(5-6): 316-326.

8. OMS. Global monitoring report on financial protection in health 2019. Geneva: World Health Organization and International Bank for Reconstruction and Development / The World Bank; 2020. Licence: CC BY-NC-SA 3.0 IGO.

9. OMS. La atención primaria de salud más necesaria que nunca. Informe sobre la salud en el mundo 2008. Informe de un Grupo Científico de la OMS; Ginebra: OMS; 2008. Serie de informes técnicos: clasificación NLM: W 84.6 ISBN 97892 43363732

10. Organización Panamericana de la Salud. Renovación de la atención primaria de salud en las Américas: documento de posición de la Organización Panamericana de la Salud. Washington, D.C.: OPS; 2007.

11. Castillo C, Manchola H, Garrafa, Volnei Cunha $T$, \& Hellmann F. El acceso a la salud como derecho humano en políticas internacionales: reflexiones críticas y desafíos contemporáneos. Ciência \& Saúde Coletiva, 2017; 22(7): 2151-
2160. 10.1590/1413-

81232017227.04472017

12. Hernández M. Sistemas universales de protecciones sociales como alternativa a la Cobertura Universal en Salud (CUS). Saúde em Debate [online]. 43 n. spe5 [Accedido 19 Enero 2021], pp. 29-43. Disponible en: 10.1590/010311042019S503>. ISSN 2358-2898. 10.1590/0103-11042019S503.

13. Perticara M. Incidencia de los gastos de bolsillo en salud en siete países latinoamericanos. Serie Políticas Sociales 141. Santiago, Chile: CEPAL; 2008.

14. Knaul FM, Wong $R$, Arreola-Ornelas $H$, Méndez O, Bitran R, Campino AC, et al. Gastos catastróficos en salud de los hogares: un análisis comparativo de doce países en América Latina y el Caribe. Salud Pública De México, 2011: 53, 95.

http://www.scielo.org.mx/scielo.php?sc ript $=$ sci_arttext\&pid=S003636342011000800005.

15. Abeldaño R A. Análisis del gasto de los hogares en salud en Argentina, como componente de la cobertura universal de salud. Ciência \& Saúde Coletiva [online]. 2017; 22(5) [Accedido 4 Abril 2021], pp. 1631-1640. Disponible en: $10.1590 / 1413-$

81232017225.21102015 . ISSN 16784561. $10.1590 / 1413-$ 81232017225.21102015.

16. Luiza VL, Tavares NUL, Oliveira MA, Arrais PSD, Ramos LR, da Silva Dal Pizzol LR, et al. Catastrophic expenditure on medicines in Brazil. Rev Saude Publica. 2016; 50(suppl 2):15s.

17. Gallegos E, Muñoz A. Proteccion financiera en salud: evolució $n$ y comparacio' n del gasto de bolsillo de los hog- ares chilenos. Rev Med Chile 2018; 146:737-44

18. Koch KJ, Cid Pedraza C, \& Schmid A. Out-of-pocket expenditure and financial protection in the chilean health care system-a systematic review. Health Policy, 2017; 121(5), 481-94. 10.1016/j.healthpol.2017.02.013

19. Pérez-Valbuena GJ, Silva-Ureña A. "Una mirada a los gastos de bolsillo en salud para Colombia," Chapters, in: Jaime Bonet \& Karelys Guzmán-Finol \& Lucas Wilfried Hahn-De-Castro (ed.), La salud en Colombia: una perspectiva regional, chapter 2017; 11: 365-404, Banco de la Republica de Colombia.

20. Alvis-Zakzuk J, Marrugo-Arnedo C, Alvis-Zakzuk NJ, Gómez de la Rosa F, Florez-Tanus A, Moreno-Ruiz D, et al. Gasto de bolsillo y gasto catastrófico en 
salud en los hogares de Cartagena, Colombia. Revista de Salud Pública [online]. 2018; 20 (5) [Accedido 4 Abril 2021], pp. 591-598. Disponible en: 10.15446/rsap.V20n5.61403 >. Epub 26 Oct 2020. ISSN 0124-0064. 10.15446/rsap.V20n5.61403.

21. Knaul FM, Arreola-Ornelas $H$, Wong $R$, Lugo-Palacios DG, Méndez-Carniado $O$. Efecto del seguro popular de salud sobre los gastos catastróficos y empobrecedores en méxico, 20042012. Salud Publica De Mexico, 2018; 60(2), 130-140. 10.21149/9064

22. Proaño Falconi D, Bernabé E. Determinants of catastrophic healthcare expenditure in Peru. International Journal of Health Economics and Management, 2018; 18(4), 425-436. 10.1007/s10754-018-9245-0

23. Pavone MP, Sánchez EJ. Determinantes del gasto de bolsillo en salud de la población pobre atendida en servicios de salud públicos en Perú, 2010-2014. Revista Panamericana De Salud Publica $=$ Pan American Journal of Public Health, 2018; 42,20. 10.26633/RPSP.2018.20

24. Gerrero G. Gasto de bolsillo en salud y riesgo de pobreza en hogares peruanos. Perú 2017. Rev. Salud \& Vida Sipanense. 2020; 7(2):27-40.

25. O'Donnell O, Van Doorslaer E, Wagstaff, A, Lindelow M. Analyzing Health Equity Using Household Survey Data: A Guide to Techniques and Their Implementation. Washington, DC: The World Bank. 2008.

26. WHO. Global Reference List of 100 Core Health Indicators (plus health-related SDGs). Geneva: World Health Organization; 2018. Licence: CC BY-NCSA 3.0 IGO.

27. World Health Organization. The World Bank. Tracking universal health coverage first global monitoring report. France. 2015. Disponible en: http://apps.who.int/iris/bitstream/handl e/10665/174536/9789241564977_eng. pdf; jsessionid $=97710$ DCFOE77797D32B 8B1F6E8CD58F1? sequence $=1$

28. WHO. The Global Observatory. Health Financing:

https://www.who.int/data/gho/data/the mes/topics/health-financing

29. Gaete R. Financiamiento y Gasto en Salud. Fundación CIRD. 2017. Disponible en: https://es.slideshare.net/rubengaete3/fi nanciamiento-y-gasto-en-salud-en-elparaguay

30. Dalal K. Evolution and patterns of global health financing 1995-2014: development assistance for health, and government, prepaid private, and outof-pocket health spending in 184 countries. The Lancet. 2017. 10.1016/S0140-6736(17)30874-7

31. OCDE. Estudio multidimensional de Paraguay: Volumen 2. Análisis detallado y recomendaciones, Caminos de Desarrollo, Éditions OCDE, Paris. 2018. 10.1787/9789264306226-es

32. Giménez $\mathrm{E}$, Flores $\mathrm{L}$, Rodríguez JC, Ocampos G, Peralta N. Gastos catastróficos de salud en los hogares del Paraguay. Mem. Inst. Investig. Cienc. Salud. 2018;16(2): 38-48.

33. Giménez E, Rodríguez JC, Ocampos G. Gasto catastrófico y cobertura universal de salud: análisis en el sistema nacional de salud del Paraguay. An. Fac. Cienc. Méd. (Asunción) / 2018; 51(3).

34. Banco Mundial. Paraguay. Revisión del gasto público en los sectores sociales. Capítulo III: Salud. (c) World Bank. 2018.

35. Giménez E, Ocampos G, Araujo JM, Peralta N, Rodríguez JC. Gastos empobrecedores de salud: un desafío para la cobertura universal de salud en el Paraguay, Anales EFACIM, 2019; 52(1): 33-42.

36. Giménez Caballero $\mathrm{E}$, Rodríguez JC, Ocampos G, Flores L. Composición del gasto de bolsillo en el sistema de salud del Paraguay. Mem. Inst. Investig. Cienc. Salud. 2017; 15(3): 64-72.

37. Benítez G. Paraguay: Distribución del Gasto en Salud y Gastos de Bolsillo. Principales resultados. CADEP. Asunción Paraguay. 2017. Disponible en: http://www.cadep.org.py/uploads/2017 106/Gastos-de-bolsillo-en-salud-OK.pdf

38. DGEEC. Principales resultados de pobreza monetaria y distribución de ingreso $2018 . \quad$ Paraguay 2018. Disponible en: https://www.dgeec.gov.py/Publicacione s/Biblioteca/POBREZA-MONETARIA\%202018/Pobreza\%20Monetaria Boletin.pdf

39. Paraguay. Ley $n^{\circ} 4.087$. De regulación de transferencias monetarias condicionadas.

40. Paraguay. Ley No 3728. Establece el derecho a la pensión alimentaria para las personas adultas mayores en situación de pobreza.

41. Paraguay. Constitución de la República del Paraguay. Disponible en: http://www.jme.gov.py/ transito/leyes/1992.html

42. Paraguay. Poder Legislativo. Ley 1032/1996. Que crea el Sistema Nacional de Salud. 1996. Disponible en: 
www.leyes.com.py/todas_disposiciones/ 1996/leyes/ley_1032_96.php

43. Paraguay. Poder Legislativo. Ley No 5099 / de la gratuidad de aranceles de las prestaciones de salud de los establecimientos del Ministerio de Salud Pública y Bienestar Social. 2013.

44. Serafini V. Pobreza en Paraguay: crecimiento económico y conflicto redistributivo. (c) Centro de Análisis y Difusión de la Economía Paraguaya, CADEP. Asunción - Paraguay. ISBN: 978-99967-937-1-4 (versión digital). 2019. Disponible en: http://www.cadep.org.py/2019/06/pobr eza-en-paraguay-crecimientoeconomico-y-conflicto-redistributivo/

45. Serafini V, Zavattiero C. (2018). La protección social en el Paraguay; avances y desafíos para la garantía de derechos. Revista MERCOSUR De Politicas Sociales, 2018; 2: 173-191. doi: $10.28917 /$ ism.2018-v2-173

46. Paraguay. Instituto Nacional de Estadísticas (INE). Bases de datos de la Encuesta Permanente de Hogares por años. Disponible en: https://www.ine.gov.py/datos/encuesta s/eph/IngFliar/ 